\title{
La Qualité des Soins Prénatals selon la Perspective des Clientes au Cameroun. (Districts de santé de Nkongsamba, Bafang et Mfou) ${ }^{1}$
}

\author{
RWENGE Mburano \\ Institut de Formation et de Recherche Démographiques, (IFORD)
}

\section{Résumé}

Etant donné que la qualité des soins détermine la demande de soins maternels et directement le risque qu'une mère décède pendant l'accouchement ou quelques jours après, en cas de complications, nous nous sommes fixé comme objectif dans cette étude d'évaluer son niveau dans le cas camerounais, en nous orientant vers la perspective des clientes. Les données utilisées ici sont celles d'une enquête que nous avons menée, en août 2005 dans le district de santé de Nkongsamba (Province du Littoral) et en janvierfévrier 2006 dans les districts de santé de Bafang (Province de l'Ouest) et Mfou (Province du Centre), auprès des femmes ayant fréquenté les services des soins prénatals au cours des six derniers mois.

Il ressort des analyses effectuées que, même si dans les milieux étudiés des problèmes importants ont été observés dans les études antérieures aux niveaux structurels et $d u$ processus des soins dans les formations sanitaires publiques et privées, les enquêtées ayant utilisé les services des soins prénatals au cours de la période de référence ci-dessus mentionnée ont été pour la plupart satisfaites de l'état des ressources physiques et des matériels, de l'organisation des services, du traitement qu'elles ont reçues pendant les consultations prénatales, etc. Toutefois, le taux de satisfaction s'est avéré, dans la plupart des cas des éléments de la qualité considérés, davantage faible chez celles ayant utilisé les formations sanitaires publiques à l'exception du cas du système de recommandation des clientes. La première conclusion qui ressort des résultats de cette étude est que les clientes tolèrent recevoir les soins prénatals de qualité moindre dans les milieux étudiés. La seconde est que le niveau de la qualité des soins prénatals varie selon le type de formations sanitaires et le district de santé. L'on devrait tenir compte de l'ensemble de ces éléments dans les politiques d'amélioration de la qualité des soins prénatals dans les milieux étudiés.

${ }^{1}$ Cette étude a été réalisée dans le cadre du projet "Evaluation de la qualité des soins obstétricaux au Cameroun. (Provinces du Centre, du Littoral et de l'Ouest)" qui a bénéficié du soutien financier de l'Organisation Mondiale de la Santé dans le cadre de son Programme Spécial de Recherche, de Développement et de Formation à la Recherche en Reproduction Humaine. 


\section{Introduction}

A la Conférence Internationale sur la Population et le Développement (CIPD) tenue à Caire en 1994, la plupart des pays africains se sont fixés comme objectif de réduire significativement le niveau de mortalité maternelle. Ce dernier est la cible de deux des huit Objectifs du Millénaire pour le Développement (OMD). On remarque cependant, dix ans plus tard, que le niveau de mortalité maternelle reste élevé et presque stable dans ces pays². Etant donné que certaines causes médicales de décès maternels peuvent être traitées (WHO, 2002), ce phénomène se justifie en partie par le taux faible de recours à l'assistance médicale pendant la grossesse et l'accouchement. D'après l'OMS, la fréquence du recours aux soins prénatals parmi les femmes enceintes est de $63 \%$ en Afrique contre 97\% en Europe, 95\% en Amérique du Nord, 73\% en Amérique Latine et Caraïbes et $65 \%$ en Asie. La fréquence du recours à l'assistance médicale pendant l'accouchement est davantage plus faible : $42 \%$ en Afrique contre $98 \%$ en Europe, $99 \%$ en Amérique du Nord, $75 \%$ en Amérique Latine et Caraïbes et $53 \%$ en Asie. Selon la même source, moins de $30 \%$ des femmes reçoivent les soins postnataux en Afrique subsaharienne contre $90 \%$ dans les pays développés. La revue de la littérature sur le sujet met en évidence deux types de facteurs explicatifs du faible recours aux soins modernes par les femmes dans les pays en développement pendant l'accouchement: les facteurs relatifs à la demande de soins et ceux relatifs à l'offre.

Dans le premier ensemble, on retrouve les facteurs culturels et économiques. Selon l'approche culturelle, la fréquentation des services de santé dépend des institutions sociales telles que les coutumes, les réseaux de solidarité, les perceptions ou représentations symboliques de la grossesse et de l'accouchement... et du degré d'ouverture à la modernité (Beninguisse, 2003). L'approche économique, quant à elle, met la fréquentation des services de santé en relation avec les conditions économiques dans lesquelles vivent les femmes (Thaddeus et Maine, 1998; Ransom et Yinger, 2002 ; Beninguisse, 2003). Dans le second ensemble, on retrouve les facteurs suivants : accessibilité des soins (en termes de disponibilité, de temps ou de coût), qualification du personnel sanitaire, moyens techniques disponibles, continuité des services, accueil des consultants, temps d'attente, etc. (Vernon et Foreit, 1999 ; Hulton et al., 2000 ; Lantis et al., 2002; Ransom et Yinger, 2002). Le niveau élevé de mortalité maternelle observé dans les pays en développement, africains en particulier, se justifie aussi par le fait que certains facteurs relatifs à

${ }^{2}$ Il s'agit d'une estimation des Nations Unies.

24 
Rwenge Mburanoe: La Qualité des Soins Prénatals selon la Perspective des Clientes au Cameroun. (Districts de santé de Nkongsamba, Bafang et Mfou)

l'offre ci-dessus mentionnés, notamment l'accessibilité aux soins obstétricaux d'urgence, la qualification du personnel sanitaire et les moyens techniques disponibles, déterminent directement le risque qu'une mère décède pendant l'accouchement ou quelques jours après, en cas de complications.

Au Cameroun, le niveau de mortalité maternelle est estimé à 430 décès pour 100.000 à partir des données de l'Enquête Démographique et de Santé de 1998. Bien que les mêmes données aient aussi révélé qu'il y a une proportion importante des femmes camerounaises qui ne recourent pas aux soins modernes pendant la grossesse et l'accouchement (respectivement $37,1 \%$ et $54,2 \%$ ), il y a peu de recherches explicatives de ce comportement aussi bien en sciences sociales (démographie, sociologie, anthropologie, etc.) qu'en épidémiologie ou en santé publique. Selon le peu de recherches sociales réalisées au Cameroun, les logiques qui sous-tendent les comportements sanitaires relatifs à la maternité dans ce pays devraient être recherchées dans une perspective globale. Ce qui témoigne de la pertinence d'évaluer la qualité de l'offre de soins prénatals dans ce pays, en s'orientant non seulement vers la perspective des prestataires mais aussi vers celles des clientes. Cette étude est orientée vers la deuxième perspective. Il s'agit en clair ici de montrer comment les clientes perçoivent les soins prénatals et d'évaluer leur degré de satisfaction par rapport à ceux-ci.

Nous présenterons d'abord ci-dessous les résultats de notre synthèse de la littérature sur la qualité des soins et le cadre conceptuel de référence et les indicateurs, ensuite les données et méthode d'analyse et enfin les résultats et la conclusion et les recommandations.

Qualité des soins maternels dans les pays en développement selon la perspective des clientes

Parmi les facteurs défavorables au recours aux services des soins modernes pendant la grossesse et l'accouchement on retrouve notamment les facteurs socioculturels et la considération selon laquelle ces services sont de qualité médiocre (Ransom et Yinger, 2002). En Russie, par exemple, les femmes ont, entre autres, considéré parmi les raisons de retarder les consultations prénatales, les soins médiocres reçus pendant les grossesses précédentes, le fait qu'elles 
passaient beaucoup de temps avant d'être reçues, la peur et les attitudes négatives des médecins (Dennis et al., 19953).

Dans une étude réalisée au Cameroun, les femmes ont cité la préservation de l'intimité, la discrétion des consultations, la mise à leur disposition du placenta, la présence réconfortante de l'entourage familial tout au long du processus de l'enfantement et les comportements des prestataires des soins parmi leurs attentes et préférences en matière des services des soins relatifs à l'accouchement (Beninguisse et al., 2003). Il n'est pas alors étonnant que les temps longs d'attente, le manque de soutien moral, la pratique simultanée de plusieurs accouchements dans la même salle, l'utilisation collective des salles de séjour et du matériel d'interventions obstétricales et la proscription ou la limitation de l'entourage familial couramment observés dans certaines maternités en Afrique puissent entraver l'utilisation des services obstétricaux. Enfin, d'autres modalités d'attitudes négatives des prestataires ont été aussi observées dans le cadre des soins obstétricaux en milieu rural à Maharashtra (en Inde) (Gupte M., Bandewar S. et Pisal H., 1999) :

"Heart attacks and childbirth can happen at any time. We must have a clinic close by. Someone can die just because of this. They make us clean up the labor room after our daughters deliver. What do they charge us for? What's the use of the nurses and ayahs [helpers]? The first childbirth hurts, doesn't it? If we scream, they shout and slap us. They say, "You didn't feel any shame when you got the thing in there. Why are you shouting when it's coming out now?" They don't give us any information. If they don't pay any attention, we're forced to keep quiet and stop asking."

La satisfaction des clientes avec les soins est associée aux attitudes et comportements positifs des prestataires (Murthy, 1999). Les clientes ont plus de risque d'être satisfaites avec les services si les prestataires les traitent avec respect et amicalement. De même, les clientes se sentent confortables lorsqu'elles sont servies en privé et sont rassurées que toutes les informations qu'elles ont données seront gardées confidentielles. La satisfaction des clientes est aussi associée à la facilité de rencontrer les médecins et aux temps courts d'attente.

Plusieurs études qualitatives sur les caractéristiques des programmes de lutte contre les IST mis en place au Sénégal, en Ethiopie, au Bénin, au Maroc et au Swaziland ont révélé que les services publics de santé sont plus concernés que d'autres par le

\footnotetext{
${ }^{3}$ Cité par l'OMS dans son étude non datée sur les priorités de recherches sur la qualité des soins.

26
} 
Rwenge Mburanoe: La Qualité des Soins Prénatals selon la Perspective des Clientes au Cameroun. (Districts de santé de Nkongsamba, Bafang et Mfou)

problème de mauvaise qualité des soins. Elles ont révélé, en effet, qu'une proportion importante des clients des services publics de santé expriment leur insatisfaction suite au fait que les prestataires n'y leur accordent pas d'intérêt, aux attitudes négatives des prestataires, à l'insuffisance du temps qu'ils leur consacrent, à l'inaptitude des premiers d'effectuer leurs examens physiques, au manque de confidentialité et d'intimité, à la mauvaise qualité des salles d'attente et aux longs temps d'attente (Field et al., 19984).

Les travaux récents sur les soins à l'avortement suggèrent que les caractéristiques des soins abortifs et post-abortifs valorisées par les clientes sont différentes de celles valorisées par elles dans d'autres services de santé reproductive. On retrouve, par exemple en Inde (Gupte et al., 1999), parmi ces caractéristiques, l'accès à un prestataire qualifié du secteur privé dans la ville voisine, l'intimité et la confidentialité chez les femmes mariées et non mariées. L'absence de l'approbation et de la signature du conjoint en faisait aussi partie chez les premières, la discrétion chez les secondes. En plus de l'intimité, le soutien et la rapidité des services étaient considérés importants chez les femmes roumaines fréquentant les hôpitaux (Johnson et al., 19965).

Cette brève revue de la littérature a révélé qu'on peut évaluer la qualité des soins selon la perspective des clientes soit à partir de leurs perceptions du processus des soins soit à partir de leurs degrés de satisfaction selon les différentes dimensions de la qualité des soins.

\section{Cadre conceptuel et indicateurs}

Le cadre conceptuel utilisé ici est celui élaboré par les chercheurs de Population Council notamment Al-Qutob et al. (1998) et Al-Qutob et al. (1996).

Ces derniers considèrent les services de santé reproductive dans un continuum des services qui commence avec la structure et s'accomplit à travers un processus. Le résultat final est l'aboutissement de ce processus.

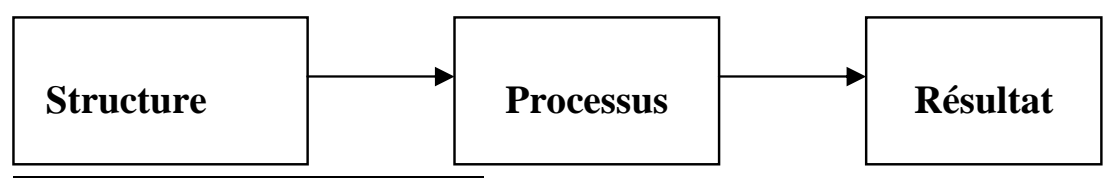

${ }^{4}$ Idem note de bas de page $n^{\circ} 3$.

${ }^{5}$ Idem note de bas de page $n^{\circ} 3$. 
Ils définissent comme suit les différents concepts en se référant à Donabedian (1980) :

- La structure: est l'ensemble des ressources humaines et des ressources financières et matérielles mises à la disposition des prestataires et le cadre physique et organisationnel dans lequel ils travaillent.

- Le processus: est l'ensemble des activités ayant lieu entre les prestataires et les clientes. Il se réfère à la transaction dans laquelle les premiers utilisent les ressources disponibles pour gérer les problèmes techniques qu'ils rencontrent et les problèmes de santé auxquels font face les secondes.

- Résultat : inclut deux éléments : l'impact direct du traitement reçu par la cliente sur sa santé actuelle et future et celle de l'enfant, et l'impact indirect sur sa satisfaction des services qui lui sont offerts.

Dans ce cadre conceptuel, les différents éléments de la qualité en relation avec les services de santé reproductive sont définis à chaque étape du continuum.

Les éléments de la qualité retenus ainsi que leurs définitions sont les suivants :

- Ressources : c'est l'ensemble des ressources humaines, physiques, matérielles et financières disponibles dans le centre de santé.

- Gestion administrative : c'est l'ensemble des activités disponibles dans le centre de santé à travers lesquelles les ressources humaines, physiques, matérielles et financières sont rationnellement utilisées pour atteindre les objectifs fixés.

- Relations entre clientes et prestataires: décrit les relations interpersonnelles entre prestataires et clientes. Celles-ci sont supposées être établies et maintenues par des prestataires courtois et compréhensifs possédant de bonnes aptitudes d'écoute et capables de soigner les femmes en privé avec respect.

- Echanges d'information: décrit le flux d'information sur la santé entre les prestataires et les clientes. La manière dont les informations sont échangées entre ces deux derniers dépend de la compétence technique des premiers et des relations entre les deux. 
Rwenge Mburanoe : La Qualité des Soins Prénatals selon la Perspective des Clientes au Cameroun. (Districts de santé de Nkongsamba, Bafang et Mfou)

- Compétence technique : se réfère aux qualifications et expériences des prestataires et aux manières dont ils utilisent leurs connaissances techniques et leurs aptitudes pour administrer des soins préventifs et curatifs aux femmes.

- Continuité : se réfère aux mécanismes qui renforcent et encouragent l'utilisation régulière des services.

Etant donné que la perspective utilisée ici pour évaluer la qualité des soins prénatals est celle des clientes, des indicateurs ont été définis uniquement selon la seconde et la troisième étapes du continuum (processus et résultat).

Tableau 1: Indicateurs des différents éléments de la qualité

\begin{tabular}{|c|c|c|}
\hline Eléments de la qualité & Processus & Résultat \\
\hline Ressources & - & $\begin{array}{l}\text { Satisfaction de clientes des } \\
\text { matérielles utilisées pour les } \\
\text { servir }\end{array}$ \\
\hline Gestion & $\begin{array}{l}\text { Risque de rentrer sans être } \\
\text { servie }\end{array}$ & $\begin{array}{l}\text { Satisfaction de clientes des } \\
\text { heures d'ouverture } \\
\text { Satisfaction de clientes de } \\
\text { l'organisation des services }\end{array}$ \\
\hline $\begin{array}{l}\text { Relations entre } \\
\text { prestataires et clientes }\end{array}$ & $\begin{array}{l}\text { Perception du temps } \\
\text { d'attente par les clientes } \\
\text { Perception de la durée des } \\
\text { consultations par les clientes } \\
\text { Attitudes des prestataires } \\
\text { pendant les consultations }\end{array}$ & - \\
\hline $\begin{array}{l}\text { Compétence technique des } \\
\text { prestataires }\end{array}$ & - & $\begin{array}{l}\text { Satisfaction de clientes des } \\
\text { soins reçus } \\
\text { Satisfaction de clientes des } \\
\text { traitements de complications } \\
\text { obstétricales }\end{array}$ \\
\hline $\begin{array}{l}\text { Echange d'information } \\
\text { entre prestataires et } \\
\text { clientes }\end{array}$ & - & $\begin{array}{l}\text { Satisfaction de clientes des } \\
\text { méthodes utilisées pour les } \\
\text { informer }\end{array}$ \\
\hline $\begin{array}{l}\text { Utilisation régulière des } \\
\text { services }\end{array}$ & $\begin{array}{l}\text { Possibilité de rencontrer le } \\
\text { même prestataire plusieurs } \\
\text { fois en cas de besoin }\end{array}$ & $\begin{array}{l}\text { Satisfaction de clientes des } \\
\text { heures de rendez-vous } \\
\text { Satisfaction de clientes des } \\
\text { méthodes utilisées pour les } \\
\text { recommander à d'autres } \\
\text { spécialistes }\end{array}$ \\
\hline
\end{tabular}

\section{Données et méthode d'analyse}

Les données utilisées ici sont issues d'une enquête que nous avons réalisée au Cameroun, en août 2005 dans le district de santé de Nkongsamba (Province du Littoral) et en janvier-février 2006 dans les 
district de santé de Bafang (Province de l'Ouest) et Mfou (Province du Centre), auprès des femmes ayant fréquenté les services publics et privés des soins prénatals au cours des six derniers mois.

A Nkongsamba, les soins prénatals étaient au moment de l'enquête offerts par sept Centres de Santé Intégrés (CSI) au premier niveau du système des soins, deux Centres Médicaux d'Arrondissement (CMA) au second niveau et un hôpital au niveau supérieur. Ils étaient aussi dans ce district de santé offerts par deux Centres de Santé Privés Confessionnels (CSPC), six Centres de Santé Privés Laïcs (CSPL) et un hôpital protestant.

A Bafang, ils étaient aussi offerts par les formations sanitaires publiques et privées. On retrouve dans le premier groupe sept CSI, deux CMA et un hôpital et dans le second un hôpital confessionnel, trois CSPC et quatre CSPL. Par rapport au premier district de santé, le système privé laïc était au moment de l'enquête moins développé ici.

A Mfou, les soins prénatals étaient offerts par huit CSI, un PMI, un dispensaire, un hôpital et cinq CSPC. Par rapport aux deux précédents districts de santé, le système privé laïc était ici absent au moment de l'enquête. Une partie de sa population fréquentait par conséquent en ce moment les formations privées laïques basées à Yaoundé (la capitale politique du pays) car la distance qui lui sépare de ce district de santé n'est que de $27 \mathrm{~km}$.

Dans l'ensemble, 1133 femmes ont été enquêtées dans les aires de santé ayant été aléatoirement sélectionnées dans les trois districts de santé. Respectivement 419, 390 et 324 femmes ont été enquêtées à Nkongsamba, Bafang et Mfou. Les données collectées portent sur les relations entre prestataires et clientes ayant fréquenté les services des soins prénatals au cours des six derniers mois, d'autres éléments du processus des soins et la satisfaction de clientes avec les soins.

Les données collectées ont été analysées en recourant aux techniques de la statistique descriptive d'analyse des tableaux à deux dimensions. Ceci a permis de mettre en évidence la variation du niveau de la qualité des soins selon le milieu et le type de formations sanitaires. 
Rwenge Mburanoe : La Qualité des Soins Prénatals selon la Perspective des Clientes au Cameroun. (Districts de santé de Nkongsamba, Bafang et Mfou)

\section{Résultats}

\section{Caractéristiques des enquêtées}

Sur le plan de l'âge, tous les groupes des femmes sont représentés dans l'échantillon dans les trois districts de santé.

Tableau 2: Répartition des enquêtées selon quelques caractéristiques socio-démographiques et le district de santé

\begin{tabular}{|c|c|c|c|c|}
\hline \multirow{2}{*}{$\begin{array}{l}\text { Caractéristiques } \\
\text { Socio -démographiques }\end{array}$} & \multicolumn{3}{|c|}{ Districts de santé } & \multirow[t]{2}{*}{ Ensemble } \\
\hline & $\begin{array}{c}\text { NKONGSA } \\
\text { MBA }\end{array}$ & $\begin{array}{c}\text { BAFAN } \\
\text { G }\end{array}$ & MFOU & \\
\hline $\mathrm{N}$ & 415 & 386 & 319 & 1120 \\
\hline $\begin{array}{cr}\text { Groupe d'âges } \\
-\quad 15-19 \\
-\quad 20-24 \\
-\quad 25-29 \\
-\quad 30-49 \\
\end{array}$ & $\begin{array}{l}10,8 \\
32,8 \\
27,7 \\
28,7\end{array}$ & $\begin{array}{l}11,4 \\
38,1 \\
27,2 \\
23,3\end{array}$ & $\begin{array}{l}30,4 \\
29,5 \\
17,6 \\
22,5\end{array}$ & $\begin{array}{l}16,6 \\
33,7 \\
24,6 \\
25,1\end{array}$ \\
\hline $\begin{array}{cl}\text { Etat matrimonial } \\
\text { - } & \text { Célibataire } \\
- & \text { Mariée } \\
- & \text { Union libre } \\
\end{array}$ & $\begin{array}{l}20,7 \\
68,7 \\
18,6 \\
\end{array}$ & $\begin{array}{l}19,8 \\
68,4 \\
11,7 \\
\end{array}$ & $\begin{array}{l}36,6 \\
26,5 \\
36,9 \\
\end{array}$ & $\begin{array}{l}24,9 \\
54,4 \\
20,7 \\
\end{array}$ \\
\hline $\begin{array}{cl}\text { Religion } & \\
- & \text { Catholique } \\
- & \text { Protestante } \\
\text { - } & \text { Autres } \\
\end{array}$ & $\begin{array}{l}53,5 \\
36,0 \\
10,5 \\
\end{array}$ & $\begin{array}{c}73,8 \\
17,5 \\
8,7 \\
\end{array}$ & $\begin{array}{c}82,1 \\
13,3 \\
4,6\end{array}$ & $\begin{array}{c}68,7 \\
23,1 \\
8,2 \\
\end{array}$ \\
\hline $\begin{array}{lll}\text { Niveau d'instruction } & \\
- & \text { Primaire } \\
- & \text { Secondaire } & \\
& 1^{\mathrm{er}} \\
& \text { degré } \\
- & \begin{array}{l}\text { Secondaire } \\
\text { degré }\end{array} & 2^{\text {nd }} \\
& \end{array}$ & $\begin{array}{l}31,5 \\
48,0 \\
20,5\end{array}$ & $\begin{array}{l}29,5 \\
52,1 \\
18,5\end{array}$ & $\begin{array}{l}31,5 \\
53,0 \\
15,6\end{array}$ & $\begin{array}{l}30,8 \\
50,8 \\
18,4\end{array}$ \\
\hline $\begin{array}{cl}\text { Activité économique } \\
- & \text { Inactive } \\
- & \text { Elèves et } \\
& \text { étudiantes } \\
- & \text { Commerce } \\
- & \text { Agriculture } \\
- & \text { Couture et } \\
& \text { coiffure } \\
- & \text { Cadres } \\
& \text { employées } \\
\end{array}$ & $\begin{array}{c}59,9 \\
6,9 \\
\\
11,3 \\
4,7 \\
10,8 \\
\\
6,4\end{array}$ & $\begin{array}{c}60,9 \\
7,9 \\
\\
10,0 \\
4,5 \\
12,9 \\
3,7\end{array}$ & $\begin{array}{c}52,5 \\
18,6 \\
\\
5,7 \\
8,8 \\
10,1 \\
\\
4,4\end{array}$ & $\begin{array}{c}58,1 \\
10,6 \\
\\
9,2 \\
5,8 \\
11,3 \\
4,9\end{array}$ \\
\hline $\begin{array}{c}\text { A déjà accouché } \\
-\quad \text { Oui } \\
-\quad \text { Non }\end{array}$ & $\begin{array}{l}76,6 \\
23,1\end{array}$ & $\begin{array}{l}70,6 \\
29,1\end{array}$ & $\begin{array}{l}64,4 \\
35,6\end{array}$ & $\begin{array}{l}71,2 \\
28,8\end{array}$ \\
\hline $\begin{array}{cc}\text { Lieu d'accouchement } \\
- & \text { Domiciles } \\
- & \text { Formations }\end{array}$ & $\begin{array}{c}1,6 \\
98,4\end{array}$ & $\begin{array}{c}1,5 \\
98,5\end{array}$ & $\begin{array}{l}18,2 \\
81,8\end{array}$ & $\begin{array}{c}5,8 \\
94,2 \\
\end{array}$ \\
\hline
\end{tabular}


African Population Studies Vol. $22 n^{\circ} 2 /$ Etude de la Population Africaine Vol. $22 N^{\circ} 2$

\begin{tabular}{|c|c|c|c|c|}
\hline \multicolumn{2}{|c|}{ sanitaires } & & & \\
\hline Parité atteinte & 10,8 & 9,8 & 12,3 & 10,9 \\
$-\quad 0$ & 27,2 & 26,7 & 30,6 & 28,0 \\
$-\quad 1$ & 33,3 & 39,2 & 28,4 & 34,1 \\
$-\quad 2-3$ & 20,7 & 17,2 & 17,7 & 18,6 \\
$-\quad 4-5$ & 8,0 & 6,6 & 11,0 & 8,4 \\
\hline
\end{tabular}

On observe aussi ici que la proportion des adolescentes est trois fois plus élevée dans le district de santé de Mfou (30,4\%) qu'à Nkongsamba $(10,8 \%)$ et Bafang (11,4 \%). En revanche, les femmes jeunes de 20-24 ans et 25-29 ans sont plus représentées dans ces deux derniers districts de santé (respectivement $60,5 \%$ et $55,3 \%$ ) que dans le premier $(47,1 \%)$. Les femmes de $30-49$ ans sont un peu plus représentées à Nkongsamba $(28,5 \%)$ que dans les deux autres districts de santé (respectivement $23,3 \%$ et $22,5 \%$ ).

Dans le cas de l'état matrimonial, on retrouve dans l'échantillon les femmes célibataires, mariées et celles vivant en union libre. La répartition des enquêtées selon cette caractéristique varie toutefois selon le district de santé. En effet, on observe que 36,6 \% des enquêtées sont célibataires dans le district de santé de Mfou alors que dans les deux autres districts leur proportion est deux fois moins élevée (19,8\% à Bafang et 20,7 \% à Nkongsamba). On observe aussi que la proportion des enquêtées vivant en union libre est plus élevée dans le premier district de santé $(36,9 \%)$ que dans les deux autres (respectivement $11,7 \%$ et $16,6 \%$ ). En revanche, la proportion des enquêtées mariées est 2 à 3 fois moins élevée dans le premier district de santé $(26,5 \%)$ que dans les deux autres (respectivement $68,4 \%$ et $62,7 \%)$.

Dans les trois districts de santé, la majorité des enquêtées sont catholiques. Mais on constate que leur proportion est beaucoup plus élevée à Bafang (73,8 \%) et Mfou (82,1 \%) qu'à Nkongsamba (53,5\%). La proportion des enquêtées protestantes est assez importante dans ce dernier district de santé $(36,0 \%)$ au contraire notamment des deux premiers (respectivement $17,5 \%$ et $13,3 \%$ ).

Sur le plan du niveau d'instruction, 30,8 \% des enquêtées sont de niveau primaire, $50,8 \%$ de niveau secondaire premier de degré et $18,4 \%$ de niveau secondaire second degré. Le niveau d'instruction ne semble pas varier selon le district de santé. La majorité des enquêtées sont inactives $(58,1 \%)$. On retrouve dans l'échantillon une faible proportion d'enquêtées élèves ou étudiantes $(10,6 \%)$, commerçantes $(9,2 \%)$, couturières ou coiffeuses $(11,3 \%)$, agricultrices $(5,8 \%)$ ou 32 
Rwenge Mburanoe : La Qualité des Soins Prénatals selon la Perspective des Clientes au Cameroun. (Districts de santé de Nkongsamba, Bafang et Mfou)

cadres et employées (4,9\%). Les enquêtées appartenant au second groupe sont toutefois 2 à 3 fois plus représentées à Mfou que dans les deux autres districts de santé. Il en est de même de celles du cinquième groupe. En revanche, celles du troisième sont plus représentées dans ces deux derniers districts de santé.

Enfin, les femmes enquêtées grandes multipares (c'est-à-dire ayant au moins 4 enfants) qui ont accouché au cours de la période de référence sont assez représentées dans les trois districts de santé.

\section{Ressources physiques et matérielles}

Dans les trois districts de santé, le taux de satisfaction est très élevé dans l'ensemble.

Tableau 3: Pourcentage de clientes anciennes qui se sont déclarées satisfaites selon les différents éléments de la qualité des soins considérés et le type de formations sanitaires dans le district de santé de Nkongsamba

\begin{tabular}{|c|c|c|c|c|c|c|}
\hline \multirow[t]{2}{*}{ Eléments de la qualité } & \multicolumn{5}{|c|}{ Type de formations sanitaires } & \multirow{2}{*}{$\begin{array}{l}\text { Ensembl } \\
\mathrm{e}\end{array}$} \\
\hline & HD & $\mathrm{HC}$ & CMA & CSI & CSPL & \\
\hline $\mathbf{N}$ & 119 & 67 & 20 & 162 & 50 & 418 \\
\hline $\begin{array}{l}\text { Ressources } \\
\text { Etat des salles d'attente } \\
\text { Etat des salles des } \\
\text { consultations } \\
\text { Etat des matériels }\end{array}$ & $\begin{array}{l}84,9 * * \\
89,1 * * \\
91,6 * *\end{array}$ & $\begin{array}{l}95,5 \\
97,0 \\
88,1\end{array}$ & $\begin{array}{l}95,0 \\
95,0 \\
100\end{array}$ & $\begin{array}{l}98,1 \\
99,4 \\
98,1\end{array}$ & $\begin{array}{l}93,5 \\
94,0 \\
95,0\end{array}$ & $\begin{array}{l}92,8 \\
95,2\end{array}$ \\
\hline $\begin{array}{l}\text { Gestion } \\
\text { Heures d'ouverture des } \\
\text { formations sanitaires } \\
\begin{array}{l}\text { Services dans } \\
\text { formations sanitaires }\end{array} \\
\end{array}$ & $\begin{array}{l}82,2 * * \\
93,2 * *\end{array}$ & $\begin{array}{l}97,0 \\
97,5\end{array}$ & $\begin{array}{l}80,0 \\
100\end{array}$ & $\begin{array}{l}95,7 \\
99,4\end{array}$ & $\begin{array}{l}90,0 \\
97,0\end{array}$ & $\begin{array}{l}90,6 \\
96,6\end{array}$ \\
\hline \begin{tabular}{lcr} 
Relations & \multicolumn{2}{r}{ entre } \\
prestataires et clientes \\
Réponses données & aux \\
questions & par & les \\
prestataires & & \\
prestion
\end{tabular} & 95,1 & 94,1 & 100 & 99,0 & 94,4 & 95,6 \\
\hline $\begin{array}{l}\text { Compétence technique } \\
\text { Soins reçus dans les } \\
\text { formations sanitaires } \\
\text { Traitements des } \\
\text { complications }\end{array}$ & $\begin{array}{c}90,7 \\
(80,8)\end{array}$ & $\begin{array}{c}91,0 \\
(71,4)\end{array}$ & $\begin{array}{l}100 \\
(100)\end{array}$ & $\begin{array}{c}95,7 \\
(84,2)\end{array}$ & $\begin{array}{l}94,0 \\
(70,0)\end{array}$ & $\begin{array}{c}93,5 \\
(80,3)\end{array}$ \\
\hline $\begin{array}{l}\text { Echanges d'information } \\
\text { Méthodes d'information } \\
\text { utilisées par les prestataires }\end{array}$ & 96,2 & 93,9 & 85,7 & 92,9 & 97,2 & 94,2 \\
\hline
\end{tabular}


African Population Studies Vol. $22 n^{\circ} 2 /$ Etude de la Population Africaine Vol. $22 N^{\circ} 2$

\begin{tabular}{|l|c|c|c|c|c|c|}
\hline $\begin{array}{l}\text { Utilisation régulière des } \\
\text { services } \\
\text { Manières utilisées pour } \\
\text { recommander les clientes }\end{array}$ & $(100)$ & $(88,9)$ & - & $(100)$ & $(66,7)$ & $(94,7)$ \\
Heures de rendez-vous & & & & & & \\
$-\quad$ Souvent & $82,9 * *$ & 93,9 & 100 & 93,4 & 91,5 & 90,6 \\
$-\quad$ Parfois & 7,2 & 4,5 & - & 3,3 & 6,4 & 4,8 \\
$-\quad$ Pas & 9,9 & 1,5 & - & 3,3 & 2,1 & 4,6 \\
\end{tabular}

** $\mathrm{p} \leq 10 \%$.

() Effectif faible.

Tableau 4: Pourcentage de clientes anciennes qui se sont déclarées satisfaites selon les différents éléments de la qualité des soins considérés et le type de formations sanitaires dans le district de santé de Bafang

\begin{tabular}{|c|c|c|c|c|c|c|}
\hline \multirow[t]{2}{*}{ Eléments de la qualité } & \multicolumn{5}{|c|}{ Type de formations sanitaires } & \multirow{2}{*}{$\begin{array}{l}\text { Ensembl } \\
\mathrm{e}\end{array}$} \\
\hline & HD & $\mathrm{HC}$ & CMA & CSI & CSPC & \\
\hline $\mathbf{N}$ & 116 & 95 & 38 & 109 & 28 & 386 \\
\hline $\begin{array}{l}\text { Ressources } \\
\text { Etat des salles d'attente } \\
\text { Etat des salles des } \\
\text { consultations } \\
\text { Etat des matériels }\end{array}$ & $\begin{array}{c}81,0^{* *} \\
93,1 \\
88,8^{* *}\end{array}$ & $\begin{array}{l}97,9 \\
98,9 \\
95,8\end{array}$ & $\begin{array}{c}84,2 \\
92,1 \\
100\end{array}$ & $\begin{array}{l}80,7 \\
93,6 \\
97,2\end{array}$ & $\begin{array}{c}96,4 \\
100 \\
100\end{array}$ & $\begin{array}{l}86,5 \\
95,1 \\
94,8\end{array}$ \\
\hline $\begin{array}{l}\text { Gestion } \\
\text { Heures d'ouverture des } \\
\text { formations sanitaires } \\
\text { Services dans les } \\
\text { formations sanitaires }\end{array}$ & $\begin{array}{l}86,2^{* *} \\
88,7^{* *}\end{array}$ & $\begin{array}{l}93,5 \\
98,8\end{array}$ & $\begin{array}{l}86,8 \\
89,5\end{array}$ & $\begin{array}{l}87,2 \\
95,4\end{array}$ & $\begin{array}{r}96,4 \\
97,4\end{array}$ & $\begin{array}{l}88,3 \\
93,0\end{array}$ \\
\hline $\begin{array}{lcr}\text { Relations } & \text { entre } \\
\text { prestataires et clientes } \\
\text { Réponses données } & \text { aux } \\
\text { questions } & \text { par } & \text { les } \\
\text { prestataires } & & \end{array}$ & 94,2 & 98,3 & 100 & 98,6 & 100 & 97,2 \\
\hline \begin{tabular}{l}
\multicolumn{3}{l}{ Compétence technique } \\
Soins reçus dans les \\
formations sanitaires \\
Traitements \\
complications
\end{tabular} & $\begin{array}{l}86,2 * * \\
(74,1)\end{array}$ & $\begin{array}{c}94,7 \\
(88,9)\end{array}$ & $\begin{array}{r}89,5 \\
(50,0)\end{array}$ & $\begin{array}{c}95,4 \\
(66,7)\end{array}$ & $\begin{array}{l}96,4 \\
(100)\end{array}$ & $\begin{array}{l}91,9 \\
75,0\end{array}$ \\
\hline 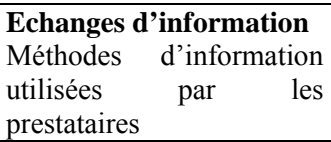 & 96,6 & 93,5 & 95,7 & 80,4 & 95,0 & 84,4 \\
\hline $\begin{array}{l}\text { Utilisation régulière des } \\
\text { services } \\
\text { Manières utilisées pour } \\
\text { recommander les clientes } \\
\text { Heures de rendez-vous } \\
\text { - Souvent } \\
\text { - Parfois } \\
\text { - Pas }\end{array}$ & $\begin{array}{c}77,4 * * \\
14,8 \\
7,8\end{array}$ & $\begin{array}{c}88,4 \\
8,4 \\
3,2\end{array}$ & $\begin{array}{c}92,1 \\
- \\
7,9\end{array}$ & $\begin{array}{c}84,8 \\
11,4 \\
3,8\end{array}$ & $\begin{array}{c}100 \\
- \\
-\end{array}$ & $\begin{array}{c}85,3 \\
9,7 \\
5,0\end{array}$ \\
\hline
\end{tabular}


Rwenge Mburanoe : La Qualité des Soins Prénatals selon la Perspective des Clientes au Cameroun. (Districts de santé de Nkongsamba, Bafang et Mfou)

$* * \mathrm{p} \leq 10 \%$.

( ) Faible effectif

Tableau 5: Pourcentage de clientes anciennes qui se sont déclarées satisfaites selon les différents éléments de la qualité des soins considérés et le type de formations sanitaires dans le district de santé Mfou

\begin{tabular}{|c|c|c|c|c|}
\hline \multirow[t]{2}{*}{ Eléments de la qualité } & \multicolumn{3}{|c|}{ Type de formations sanitaires } & \multirow{2}{*}{$\begin{array}{c}\text { Ensembl } \\
\mathrm{e}\end{array}$} \\
\hline & HD & CSI & CSPC & \\
\hline $\mathbf{N}$ & 159 & 31 & 123 & 315 \\
\hline Ressources & & & & \\
\hline Etat des salles d'attente & $90,6 * *$ & 80,6 & 97,6 & 92,1 \\
\hline Etat des salles des consultations & $96,2 * *$ & 87,5 & 96,7 & 95,2 \\
\hline Etat des matériels & 91,8 & 84,8 & 95,9 & 92,7 \\
\hline $\begin{array}{l}\text { Gestion } \\
\text { Heures d'ouverture des formations } \\
\text { sanitaires }\end{array}$ & $89,9 * *$ & 81,8 & 94,2 & 90,8 \\
\hline Services dans les formations sanitaires & $93,1 * *$ & 100 & 100 & 95,9 \\
\hline $\begin{array}{l}\text { Relations entre prestataires et clientes } \\
\text { Réponses données aux questions par les } \\
\text { prestataires }\end{array}$ & 99,0 & 100 & 94,8 & 97,7 \\
\hline $\begin{array}{l}\text { Compétence technique } \\
\text { Soins reçus dans les formations sanitaires } \\
\text { Traitements des complications }\end{array}$ & $\begin{array}{c}89,9 \\
(86,2)\end{array}$ & $\begin{array}{c}97,0 \\
(87,5)\end{array}$ & $\begin{array}{c}95,1 \\
(78,9)\end{array}$ & $\begin{array}{c}92,7 \\
(84,2)\end{array}$ \\
\hline $\begin{array}{l}\text { Echanges d'information } \\
\text { Méthodes d'information utilisées par les } \\
\text { prestataires }\end{array}$ & 95,8 & 91,7 & 94,7 & 95,2 \\
\hline $\begin{array}{l}\text { Utilisation régulière des services } \\
\text { Manières utilisées pour recommander les } \\
\text { clientes } \\
\text { Heures de rendez-vous }\end{array}$ & $(88,5)$ & $(88,9)$ & $(90,0)$ & 88,9 \\
\hline $\begin{array}{ll}\text { - } & \text { Souvent } \\
\text { - } & \text { Parfois } \\
- & \text { Pas }\end{array}$ & $\begin{array}{c}85,4 \\
12,1 \\
2,5\end{array}$ & $\begin{array}{c}96,9 \\
3,1 \\
-\end{array}$ & $\begin{array}{c}92,7 \\
4,1 \\
3,3\end{array}$ & $\begin{array}{c}89,5 \\
8,0 \\
2,5\end{array}$ \\
\hline
\end{tabular}

$* * \mathrm{p} \leq 10 \%$.

( ) Effectif faible.

On observe toutefois que ce taux varie selon le type de formations sanitaires dans le district de santé de Nkongsamba dans les cas notamment de l'état de la salle d'attente, de celui de la salle des consultations et de celui des matériels. Dans le district de Bafang, il varie dans le premier et dernier cas et dans celui de Mfou dans les deux premiers cas. 
Dans le premier district de santé, la proportion des enquêtées qui se sont déclarées satisfaites de l'état des salles d'attente et de celui de salles des consultations est moins élevée chez celles ayant été servies à l'hôpital de district (84,9 \% et $89,1 \%$ ) que chez celles ayant été servies à l'hôpital confessionnel (95,5\% et 97,0 \%) et dans les centres de santé $(95,0 \%$ dans les CMA, 98,1 \% et 99,4\% dans les CSI et $93,5 \%$ et $94,0 \%$ dans les CSPL). A Bafang, celles ayant été servies dans les formations sanitaires publiques $(81,0 \%$ à l'hôpital, 84,2 \% dans les CMA et 80,7 \% dans les CSI) se démarquent nettement des autres (97,9 \% à l'hôpital et 96,4 \% dans les CSPC) dans le cas de l'état des salles d'attente. A Mfou, le taux de satisfaction est de même dans ce dernier cas plus faible chez celles ayant été servies dans les centres de santé publics $(80,6 \%$ ) que dans les centres de santé privés $(96,2 \%)$. Le taux de satisfaction chez celles ayant été servies à l'hôpital de district $(90,6 \%)$ se situe entre les deux.

La proportion des enquêtées qui se sont déclarées satisfaites de l'état des matériels est à Nkongsamba moins élevée chez celles ayant été servies dans les hôpitaux (91,6 \% à l'hôpital de district et $88,1 \%$ à l'hôpital confessionnel) que chez d'autres (100 \% dans les CMA, 98,1 \% dans les CSI et 95,0 \% dans les CSPL). A Bafang, elle est moins élevée chez celles ayant été à l'hôpital de district $(88,8 \%)$ que chez d'autres (95,8 \% à l'hôpital confessionnel, 100 \% dans les CMA, 97,2 \% dans les CSI et $100 \%$ dans les CSPL).

En résumé, dans les trois districts de santé, les formations sanitaires publiques sont plus concernées que d'autres par la faible qualité des ressources physiques et des matériels.

\section{Gestion}

La proportion des clientes ayant déclaré être déjà rentrées sans être servies est faible dans les trois districts de santé mais à Nkongsamba elle est significativement plus élevée à l'hôpital de district $(28 \%)$ que dans les CSPL (2\%), les CSI (2,5\%), l'hôpital confessionnel ( $3 \%)$ et les CMA (10\%).

Les taux de satisfaction par rapport aux heures d'ouverture et à l'organisation des services dans les formations sanitaires sont très élevés dans les trois districts de santé. On observe aussi que ces taux varient selon le type de formations sanitaires dans chaque district de santé. 
Rwenge Mburanoe : La Qualité des Soins Prénatals selon la Perspective des Clientes au Cameroun. (Districts de santé de Nkongsamba, Bafang et Mfou)

A Nkongsamba, par rapport aux heures d'ouverture, les taux de satisfaction les moins élevés se retrouvent chez les femmes ayant été servies dans les formations publiques, à l'hôpital de district $(82,2$ $\%$ ) et dans les CMA (80,0 \%). On observe le même schéma dans les deux autres districts de santé. Par rapport à l'organisation des services, les taux de satisfaction les moins élevés se retrouvent aussi chez ces femmes dans les trois districts de santé (le milieu hospitalier étant le plus concerné à Nkongsamba et Mfou alors qu'à Bafang quelques centres de santé sont aussi concernés).

Ainsi, dans les milieux étudiés, les formations sanitaires publiques sont plus concernées que d'autres par le problème de mauvaise gestion.

\section{Relations entre prestataires et clientes}

\section{1\%) Temps d'attente}

Le temps passé dans la salle d'attente paraît long à 22,2\% des enquêtées à Nkongsamba, à 30,6 \% à Bafang et à 34,5\% à Mfou (tableaux 6, 7 et 8 ).

Tableau 6: Opinions de clientes anciennes sur la qualité des soins et leurs déclarations relatives aux attitudes des prestataires selon le type de formations sanitaires dans le district de santé de Nkongsamba

\begin{tabular}{|c|c|c|c|c|c|c|}
\hline \multirow[t]{2}{*}{ Eléments de la qualité } & \multicolumn{5}{|c|}{ Type de formations sanitaires } & \multirow{2}{*}{$\begin{array}{l}\text { Ensembl } \\
\mathrm{e}\end{array}$} \\
\hline & HD & $\mathrm{HC}$ & CMA & CSI & CSPL & \\
\hline $\mathbf{N}$ & 119 & 67 & 20 & 162 & 50 & 418 \\
\hline \multicolumn{7}{|l|}{ Gestion } \\
\hline $\begin{array}{l}\text { Etait plusieurs fois rentrée } \\
\text { sans être servie } \\
-\quad \text { Oui } \\
-\quad \text { Non }\end{array}$ & $\begin{array}{l}28,0^{* *} \\
72,0\end{array}$ & $\begin{array}{r}3,0 \\
97,0\end{array}$ & $\begin{array}{l}10,0 \\
90,0\end{array}$ & $\begin{array}{c}2,5 \\
97,5\end{array}$ & $\begin{array}{c}2,0 \\
98,0\end{array}$ & $\begin{array}{l}10,1 \\
89,9\end{array}$ \\
\hline $\begin{array}{l}\text { Relations entre prestataires } \\
\text { et clientes }\end{array}$ & & & & & & \\
\hline \begin{tabular}{cl}
\multicolumn{2}{l}{ Temps d'attente } \\
- & Long \\
- & Court \\
- & Convenable
\end{tabular} & $\begin{array}{c}42,9 * * \\
13,4 \\
43,7\end{array}$ & $\begin{array}{l}14,9 \\
13,4 \\
71,6\end{array}$ & $\begin{array}{l}15,0 \\
45,0 \\
40,0\end{array}$ & $\begin{array}{l}14,2 \\
17,3 \\
68,5\end{array}$ & $\begin{array}{l}12,0 \\
36,0 \\
52,0\end{array}$ & $\begin{array}{l}22,2 \\
19,1 \\
58,6\end{array}$ \\
\hline $\begin{array}{cl}\text { Durée des consultations } \\
- & \text { Longue } \\
- & \text { Courte } \\
- & \text { Convenable } \\
\end{array}$ & $\begin{array}{c}16,9 * * \\
16,9 \\
66,1 \\
\end{array}$ & $\begin{array}{l}13,4 \\
11,9 \\
74,6 \\
\end{array}$ & $\begin{array}{l}10,0 \\
35,0 \\
55,0 \\
\end{array}$ & $\begin{array}{l}13,0 \\
30,5 \\
76,5 \\
\end{array}$ & $\begin{array}{l}22,0 \\
24,0 \\
54,0 \\
\end{array}$ & $\begin{array}{l}15,1 \\
15,3 \\
69,5 \\
\end{array}$ \\
\hline $\begin{array}{l}\text { Consultations étaient } \\
\text { confidentielles }\end{array}$ & & & & & & \\
\hline
\end{tabular}


African Population Studies Vol. $22 n^{\circ} 2 /$ Etude de la Population Africaine Vol. $22 N^{\circ} 2$

\begin{tabular}{|c|c|c|c|c|c|c|}
\hline $\begin{array}{ll}- & \text { Oui } \\
- & \text { Non }\end{array}$ & $\begin{array}{l}88,0 \\
12,0\end{array}$ & $\begin{array}{l}89,6 \\
10,4\end{array}$ & $\begin{array}{r}95,0 \\
5,0\end{array}$ & $\begin{array}{r}92,6 \\
7,4\end{array}$ & $\begin{array}{l}90,0 \\
10,0\end{array}$ & $\begin{array}{r}90,6 \\
9,4\end{array}$ \\
\hline $\begin{array}{cc}\text { Prestataires étaient attentifs } \\
-\quad \text { Oui } \\
-\quad \text { Non }\end{array}$ & $\begin{array}{c}92,4 * * \\
7,6\end{array}$ & $\begin{array}{r}97,0 \\
3,0\end{array}$ & $\begin{array}{r}95,0 \\
5,0\end{array}$ & $\begin{array}{r}99,4 \\
0,6\end{array}$ & $\begin{array}{r}94,0 \\
6,0\end{array}$ & $\begin{array}{r}96,2 \\
3,8\end{array}$ \\
\hline \begin{tabular}{cl}
\multicolumn{2}{l}{ Prestataires étaient courtois } \\
- & Toujours \\
- & Parfois \\
- & Pas du tout
\end{tabular} & $\begin{array}{c}88,1 \\
10,2 \\
1,7\end{array}$ & $\begin{array}{l}85,1 \\
14,9 \\
-\end{array}$ & $\begin{array}{r}90,0 \\
10,0 \\
-\end{array}$ & $\begin{array}{c}93,2 \\
6,2 \\
0,6\end{array}$ & $\begin{array}{c}90,0 \\
8,0 \\
2,0\end{array}$ & $\begin{array}{c}89,9 \\
9,1 \\
1,0\end{array}$ \\
\hline $\begin{array}{l}\text { Prestataires vous laissaient } \\
\text { poser vos questions pendant } \\
\text { les consultations } \\
-\quad \text { Oui } \\
\text { - Non }\end{array}$ & $\begin{array}{r}98,8 \\
1,2\end{array}$ & 100 & $\begin{array}{c}100 \\
-\end{array}$ & $\begin{array}{c}100 \\
-\end{array}$ & $\begin{array}{r}94,7 \\
5,3\end{array}$ & $\begin{array}{r}98,9 \\
1,1\end{array}$ \\
\hline Echanges d'information & & & & & & \\
\hline $\begin{array}{l}\text { Avait entièrement reçu } \\
\text { l'information qu'elle } \\
\text { cherchait } \\
-\quad \text { Oui } \\
-\quad \text { Non }\end{array}$ & $\begin{array}{l}82,9 \\
17,1\end{array}$ & $\begin{array}{c}92,0 \\
8,0\end{array}$ & $\begin{array}{c}92,3 \\
7,7\end{array}$ & $\begin{array}{c}92,2 \\
7,8\end{array}$ & $\begin{array}{l}83,3 \\
16,7\end{array}$ & $\begin{array}{l}88,5 \\
11,5\end{array}$ \\
\hline $\begin{array}{l}\text { Utilisation régulière des } \\
\text { services } \\
\text { Quand elle voulait elle } \\
\text { pouvait rencontrer un même } \\
\text { prestataire plusieurs fois } \\
\text { - Toujours } \\
\text { - Parfois } \\
\text { - Pas du tout }\end{array}$ & $\begin{array}{c}66,7 \\
29,2 \\
4,2\end{array}$ & $\begin{array}{c}74,2 \\
19,4 \\
6,4\end{array}$ & $\begin{array}{r}81,8 \\
18,2 \\
-\end{array}$ & $\begin{array}{c}73,9 \\
18,5 \\
7,6\end{array}$ & $\begin{array}{c}80,6 \\
12,9 \\
6,5\end{array}$ & $\begin{array}{c}73,0 \\
21,1 \\
5,9\end{array}$ \\
\hline
\end{tabular}

$* * \mathrm{p} \leq 10 \%$.

Tableau 7: Opinions de clientes anciennes sur la qualité des soins et leurs déclarations relatives aux attitudes des prestataires selon le type de formations sanitaires dans le district de santé de Bafang

\begin{tabular}{|c|c|c|c|c|c|c|}
\hline \multirow[t]{2}{*}{ Eléments de la qualité } & \multicolumn{5}{|c|}{ Type de formations sanitaires } & \multirow[t]{2}{*}{ Ensemble } \\
\hline & HD & $\mathrm{HC}$ & CMA & CSI & CSPC & \\
\hline $\mathbf{N}$ & 116 & 95 & 38 & 109 & 28 & 386 \\
\hline Gestion & & & & & & \\
\hline $\begin{array}{l}\text { Etait plusieurs fois rentrée } \\
\text { sans être servie } \\
-\quad \text { Oui } \\
-\quad \text { Non }\end{array}$ & $\begin{array}{c}8,6 \\
91,4\end{array}$ & $\begin{array}{c}9,6 \\
90,4\end{array}$ & $\begin{array}{c}7,9 \\
92,1\end{array}$ & $\begin{array}{l}12,0 \\
88,0\end{array}$ & $\begin{array}{l}17,9 \\
82,1\end{array}$ & $\begin{array}{l}10,4 \\
89,6\end{array}$ \\
\hline $\begin{array}{l}\text { Relations entre prestataires } \\
\text { et clientes }\end{array}$ & & & & & & \\
\hline \begin{tabular}{cl}
\multicolumn{2}{l}{ Temps d'attente } \\
- & Long \\
- & Court \\
- & Convenable
\end{tabular} & $\begin{array}{c}38,8 * * \\
16,4 \\
44,8\end{array}$ & $\begin{array}{l}24,2 \\
18,9 \\
56,8\end{array}$ & $\begin{array}{l}21,1 \\
34,2 \\
44,7\end{array}$ & $\begin{array}{l}33,9 \\
17,4 \\
48,6\end{array}$ & $\begin{array}{l}17,9 \\
46,4 \\
35,7\end{array}$ & $\begin{array}{l}30,6 \\
21,2 \\
48,2\end{array}$ \\
\hline
\end{tabular}


Rwenge Mburanoe : La Qualité des Soins Prénatals selon la Perspective des Clientes au Cameroun. (Districts de santé de Nkongsamba, Bafang et Mfou)

\begin{tabular}{|c|c|c|c|c|c|c|}
\hline $\begin{array}{cl}\text { Durée des consultations } \\
- & \text { Longue } \\
- & \text { Courte } \\
- & \text { Convenable } \\
\end{array}$ & $\begin{array}{l}13,9 \\
18,3 \\
67,8\end{array}$ & $\begin{array}{l}11,6 \\
13,7 \\
74,7\end{array}$ & $\begin{array}{l}13,5 \\
18,9 \\
67,6\end{array}$ & $\begin{array}{l}15,0 \\
22,4 \\
62,6\end{array}$ & $\begin{array}{l}10,7 \\
25,0 \\
64,3\end{array}$ & $\begin{array}{l}13,4 \\
18,8 \\
67,8\end{array}$ \\
\hline $\begin{array}{l}\text { Consultations étaient } \\
\text { confidentielles } \\
-\quad \text { Oui } \\
-\quad \text { Non }\end{array}$ & $\begin{array}{c}89,7 * * \\
10,3\end{array}$ & $\begin{array}{l}87,4 \\
12,6\end{array}$ & $\begin{array}{l}68,4 \\
31,6\end{array}$ & $\begin{array}{l}74,4 \\
25,6\end{array}$ & $\begin{array}{c}92,9 \\
7,1\end{array}$ & $\begin{array}{l}85,8 \\
14,2\end{array}$ \\
\hline $\begin{array}{c}\text { Prestataires étaient attentifs } \\
-\quad \text { Oui } \\
-\quad \text { Non }\end{array}$ & $\begin{array}{c}93,1^{* *} \\
6,9\end{array}$ & $\begin{array}{c}98,9 \\
1,1\end{array}$ & $\begin{array}{c}92,1 \\
7,9\end{array}$ & $\begin{array}{c}98,2 \\
1,8\end{array}$ & $\begin{array}{c}100 \\
-\end{array}$ & $\begin{array}{c}96,4 \\
3,6\end{array}$ \\
\hline $\begin{aligned} & \text { Prestataires étaient courtois } \\
&- \text { Toujours } \\
&- \text { Parfois } \\
&- \text { Pas du tout }\end{aligned}$ & $\begin{array}{c}95,7 * * \\
4,3 \\
-\end{array}$ & $\begin{array}{c}95,8 \\
3,2 \\
1,1\end{array}$ & $\begin{array}{c}84,2 \\
15,8 \\
-\end{array}$ & $\begin{array}{c}99,1 \\
0,9 \\
-\end{array}$ & $\begin{array}{c}100 \\
- \\
-\end{array}$ & $\begin{array}{c}95,9 \\
3,9 \\
0,3\end{array}$ \\
\hline $\begin{array}{l}\text { Prestataires vous laissaient } \\
\text { poser vos questions pendant } \\
\text { les consultations } \\
\text { - Oui } \\
\text { - Non }\end{array}$ & $\begin{array}{c}100 \\
-\end{array}$ & $\begin{array}{c}100 \\
-\end{array}$ & $\begin{array}{c}100 \\
-\end{array}$ & 100 & 100 & $\begin{array}{c}100 \\
-\end{array}$ \\
\hline Echanges d'information & & & & & & \\
\hline $\begin{array}{l}\text { Avait entièrement reçu } \\
\text { l'information qu'elle } \\
\text { cherchait } \\
\begin{array}{c}\text { - Oui } \\
\text { - Non }\end{array}\end{array}$ & $\begin{array}{c}83,9 \\
6,1\end{array}$ & $\begin{array}{c}80,4 \\
9,6\end{array}$ & $\begin{array}{c}95,7 \\
4,3\end{array}$ & $\begin{array}{l}80,4 \\
19,6\end{array}$ & $\begin{array}{c}95,0 \\
5,0\end{array}$ & $\begin{array}{l}84,4 \\
15,6\end{array}$ \\
\hline $\begin{array}{l}\text { Utilisation régulière des } \\
\text { services } \\
\text { Quand elle voulait elle } \\
\text { pouvait rencontrer un même } \\
\text { prestataire plusieurs fois } \\
\text { - Toujours } \\
\text { - Parfois } \\
\text { - Pas du tout }\end{array}$ & $\begin{array}{c}82,5 \\
12,5 \\
5,0\end{array}$ & $\begin{array}{c}85,0 \\
13,3 \\
1,7\end{array}$ & $\begin{array}{c}66,7 \\
23,8 \\
9,5\end{array}$ & $\begin{array}{c}83,1 \\
15,6 \\
1,3\end{array}$ & $\begin{array}{c}95,5 \\
4,5 \\
-\end{array}$ & $\begin{array}{c}83,1 \\
13,8 \\
3,1\end{array}$ \\
\hline
\end{tabular}

$* * \mathrm{p} \leq 10 \%$.

Tableau 8: Opinions de clientes anciennes sur la qualité des soins et leurs déclarations relatives aux attitudes des prestataires selon le type de formations sanitaires dans le district de santé de Mfou

\begin{tabular}{|l|c|c|c|c|}
\hline \multirow{2}{*}{ Eléments de la qualité } & \multicolumn{3}{|c|}{$\begin{array}{c}\text { Types de formations } \\
\text { sanitaires }\end{array}$} & \multirow{2}{*}{ Ensemble } \\
\cline { 2 - 4 } & HD & CSI & CSPC & \\
\hline N & 159 & 31 & 123 & 315 \\
\hline Gestion & & & & \\
& & & & \\
Etait plusieurs fois rentrée sans être servie & & & & \\
$\quad \quad$ Oui & 8,2 & 9,1 & 6,5 & 7,6 \\
$-\quad$ Non & 91,8 & 90,9 & 93,5 & 92,4 \\
\hline Relations entre prestataires et clientes & & & & \\
& & & & \\
\hline
\end{tabular}


African Population Studies Vol. $22 n^{\circ} 2 /$ Etude de la Population Africaine Vol. $22 N^{\circ} 2$

\begin{tabular}{|c|c|c|c|c|}
\hline \begin{tabular}{cl}
\multicolumn{2}{l}{ Temps d'attente } \\
- & Long \\
- & Court \\
- & Convenable
\end{tabular} & $\begin{array}{l}43,4^{* *} \\
17,6 \\
39,0\end{array}$ & $\begin{array}{l}15,2 \\
63,6 \\
21,2\end{array}$ & $\begin{array}{l}28,7 \\
36,1 \\
35,2\end{array}$ & $\begin{array}{l}34,5 \\
29,7 \\
35,8\end{array}$ \\
\hline $\begin{array}{cl}\text { Durée des consultations } \\
- & \text { Longue } \\
- & \text { Courte } \\
- & \text { Convenable } \\
\end{array}$ & $\begin{array}{c}10,1 * * \\
37,7 \\
52,2\end{array}$ & $\begin{array}{l}15,2 \\
51,5 \\
33,3\end{array}$ & $\begin{array}{c}9,8 \\
37,4 \\
52,8\end{array}$ & $\begin{array}{l}10,4 \\
39,1 \\
50,5\end{array}$ \\
\hline $\begin{array}{c}\text { Consultations étaient confidentielles } \\
-\quad \text { Oui } \\
-\quad \text { Non }\end{array}$ & $\begin{array}{c}95,6^{* * *} \\
4,4\end{array}$ & $\begin{array}{l}87,9 \\
12,1\end{array}$ & $\begin{array}{c}97,6 \\
2,4\end{array}$ & $\begin{array}{c}95,6 \\
4,4\end{array}$ \\
\hline $\begin{array}{c}\text { Prestataires étaient attentifs } \\
-\quad \text { Oui } \\
-\quad \text { Non }\end{array}$ & $\begin{array}{c}95,6 \\
4,4\end{array}$ & $\begin{array}{c}100 \\
-\end{array}$ & $\begin{array}{c}97,6 \\
2,4\end{array}$ & $\begin{array}{c}96,8 \\
3,2\end{array}$ \\
\hline $\begin{array}{cl}\text { Prestataires étaient courtois } \\
- & \text { Toujours } \\
- & \text { Parfois } \\
- & \text { Pas du tout }\end{array}$ & $\begin{array}{l}73,0^{* *} \\
26,4 \\
0,6\end{array}$ & $\begin{array}{c}100 \\
- \\
-\end{array}$ & $\begin{array}{c}91,1 \\
8,1 \\
0,8\end{array}$ & $\begin{array}{c}83,0 \\
16,4 \\
0,6\end{array}$ \\
\hline $\begin{array}{l}\text { Prestataires vous laissaient poser vos } \\
\text { questions pendant les consultations } \\
\text { - Oui } \\
\text { - Non }\end{array}$ & $\begin{array}{c}97,1 \\
2,9\end{array}$ & $\begin{array}{c}93,8 \\
6,2\end{array}$ & $\begin{array}{c}96,7 \\
3,3\end{array}$ & $\begin{array}{c}96,6 \\
3,4\end{array}$ \\
\hline Echanges d'information & & & & \\
\hline $\begin{array}{l}\text { Avait entièrement } \text { reçu l'information } \\
\text { qu'elle cherchait } \\
\text { - Oui } \\
\text { - Non }\end{array}$ & $\begin{array}{c}95,7 \\
4,3\end{array}$ & $\begin{array}{c}92,3 \\
7,7\end{array}$ & $\begin{array}{c}94,7 \\
5,3\end{array}$ & $\begin{array}{c}95,2 \\
4,8\end{array}$ \\
\hline $\begin{array}{l}\text { Utilisation régulière des services } \\
\text { Quand elle voulait elle pouvait rencontrer } \\
\text { un même prestataire plusieurs fois } \\
\begin{array}{ll}\text { - } & \text { Toujours } \\
\text { - } & \text { Parfois } \\
\text { - } & \text { Pas du tout }\end{array}\end{array}$ & $\begin{array}{c}62,7 * * \\
19,6 \\
17,6\end{array}$ & $\begin{array}{c}83,3 \\
12,5 \\
4,2\end{array}$ & $\begin{array}{c}68,9 \\
28,9 \\
2,2\end{array}$ & $\begin{array}{c}69,4 \\
21,5 \\
9,1\end{array}$ \\
\hline
\end{tabular}

$* * \mathrm{p} \leq 10 \%$

Dans les deux premiers districts de santé, lorsqu'on ne considère que les hôpitaux, la proportion des femmes pour qui le temps d'attente paraît long est plus élevée chez celles ayant été servies à l'hôpital de district (42,2 \% et 36,8 \%) qu'à l'hôpital confessionnel $(14,9 \%$ et $24,2 \%)$. Lorsqu' on ne considère que les centres de santé, on n'observe pas de différence entre les centres publics et privés dans le premier district de santé au contraire notamment du second où cette proportion est davantage élevée dans les premiers (surtout dans les CSI : 33,9\%) que dans les seconds $(17,9$ $\%)$. A Mfou, on observe plutôt que cette proportion est davantage élevée dans les seconds (28,7\%) que dans les premiers (15,2\%). Dans chacun des trois districts de santé, cette proportion est plus élevée à l'hôpital de district que dans les autres formations sanitaires. 
Rwenge Mburanoe: La Qualité des Soins Prénatals selon la Perspective des Clientes au Cameroun. (Districts de santé de Nkongsamba, Bafang et Mfou)

Ce qu'on retient ici est que, dans les trois districts de santé, le temps passé dans la salle d'attente est jugé long par un nombre important de femmes surtout parmi celles ayant été consultées à l'hôpital de district.

\section{$2^{\circ}$ ) Durée des consultations}

La proportion des enquêtées pour qui la durée des consultations paraît courte est faible dans les trois districts de santé mais elle est plus élevée à Mfou (39,1 \%) qu'à Nkongsamba (15,3\%) et Bafang (18,8 \%). Dans les deux premiers districts de santé, les proportions les plus élevées se retrouvent chez les femmes ayant été servies dans les formations sanitaires publiques alors que dans l'autre on n'observe pas de variation significative selon le type de formations sanitaires.

Il ressort aussi de résultats que, dans les deux premiers districts de santé, la proportion des femmes pour qui la durée des consultations paraît courte est plus faible en milieu hospitalier que dans les centres de santé. En effet, à Nkongsamba, cette proportion est de $16,9 \%$ à l'hôpital de district alors que dans les CMA $(35,0$ \%) et CSI $(30,5 \%)$ elle est 2 fois plus élevée. A Mfou, elle est de $37,7 \%$ à l'hôpital de district et de 51,5 \% dans les CSI.

Toutes ces observations confortent l'idée selon laquelle, les formations sanitaires publiques, particulièrement les centres de santé, sont plus concernées par le problème de consultations rapides qui, souvent, sont mal perçues par les femmes enceintes.

\section{$\left.3^{\circ}\right) \quad$ Attitudes des prestataires}

La proportion des enquêtées qui pensent que les consultations étaient confidentielles est élevée dans l'ensemble mais beaucoup plus à Mfou (95,6 \%) et Nkongsamba (90,6 \%) qu'à Bafang $(80,8 \%)$. Dans le second district de santé, cette proportion ne semble pas varier selon le type de formations sanitaires au contraire notamment des deux autres districts de santé. Cette proportion est dans ces deux derniers davantage faible dans les centres de santé publics que privés confessionnels. En milieu hospitalier (public ou privé), la confidentialité est très respectée, comme dans ces derniers.

La proportion des femmes qui pensent que les prestataires étaient attentifs pendant les entretiens est très élevée dans les trois districts de santé (96,2 \% à Nkongsamba, 96,4 \% à Bafang et 96,8 \% à 
Mfou). Dans les deux premiers districts de santé, où cette proportion varie significativement selon le type de formations sanitaires, c'est dans les formations sanitaires publiques (précisément à l'hôpital de district et dans les CMA) qu'elle est inférieure à la moyenne globale. La proportion des femmes qui pensent que les prestataires étaient toujours courtois pendant les consultations est aussi élevée mais plus à Nkongsamba (95,9\%) et Bafang (90,9\%) qu'à Mfou (80,0 \%). Dans ces deux derniers districts de santé, où une variation significative est observée, sa valeur la plus faible se retrouve dans les formations sanitaires publiques, dans les CMA à Bafang, à l'hôpital de district à Mfou.

Finalement, dans les trois districts de santé, presque toutes les enquêtées ont déclaré que les prestataires leur permettaient de poser les questions qu'elles avaient pendant les consultations. Le taux de satisfaction par rapport aux réponses données par les prestataires est très élevé dans les trois milieux $(96,6 \%$ à Nkongsamba, 97,2 \% à Bafang et 97,7 \% à Mfou).

On retient de ce qui précède que les centres de santé publics sont dans les districts de santé de Bafang et Mfou plus concernés que d'autres par le problème de non respect de la confidentialité. Ils sont aussi dans le premier district de santé plus concernés que d'autres par le problème de manque de courtoisie. Dans le second district de santé, c'est le milieu hospitalier public qui est davantage concerné par le second problème.

\section{Compétence technique des prestataires}

Le taux de satisfaction par rapport aux soins reçus dans les formations sanitaires est élevé à Nkongsamba (93,5\%), Bafang (91,9 $\%)$ et Mfou (93,5\%). On observe toutefois dans le second district de santé que ce taux est significativement plus faible chez les femmes ayant été servies à l'hôpital de district $(83,2 \%)$ qu'à l'hôpital confessionnel $(94,5 \%)$ et dans les centres de santé $(92,5 \%$ dans les CMA, 95,4 \% dans les CSI et 96,4\% dans les CSPC).

Le taux de satisfaction par rapport au traitement des complications est aussi élevé dans les trois districts de santé (respectivement $80,3 \%, 75 \%$ et $84,2 \%$ ) mais on constate qu'il est plus faible que celui correspondant aux soins reçus, particulièrement à Bafang.

En définitive, le système et les techniques de gestion des complications obstétricales utilisés dans le district de santé de Bafang sont moins satisfaisants que ceux utilisés dans les deux autres districts. 
Rwenge Mburanoe: La Qualité des Soins Prénatals selon la Perspective des Clientes au Cameroun. (Districts de santé de Nkongsamba, Bafang et Mfou)

\section{Echanges d'information entre prestataires et clientes}

La proportion des enquêtées ayant déclaré avoir entièrement reçu les informations qu'elles cherchaient est élevée dans les trois districts de santé mais davantage à Mfou (95,2 \%) qu'à Bafang (84,4 $\%)$ et Nkongsamba $(85,5 \%)$.

Le taux de satisfaction par rapport aux méthodes par lesquelles elles ont été informées est très élevé (respectivement 94,2 \%, $95,2 \%$ et $95,2 \%$ ).

Ainsi, étant donné le problème d'abandon des soins obstétricaux qui se pose dans le district de santé de Mfou, les prestataires s'y donnent davantage à l'information des clientes en santé maternelle que dans les deux autres districts de santé.

\section{Utilisation régulière des services}

Parmi les femmes qui voulaient rencontrer les mêmes prestataires plusieurs fois, la proportion de celles ayant pu les rencontrer est plus élevée à Bafang (83,1 \%) qu'à Mfou (69,4\%) et Nkongsamba (73 \%). Dans le premier district de santé, cette proportion est moins élevée dans les centres de santé publics $(66,7 \%$ dans les CMA et 83,9 \% dans les CSI) que privées (95,5\%) alors qu'à Mfou on observe le schéma contraire (83,3 \% contre $68,9 \%)$. A Nkongsamba, il n'y a pas dans l'ensemble de variation significative. Mais on observe toutefois dans ce district de santé, comme dans le précédent, que cette proportion est davantage faible à l'hôpital de district (respectivement $66,7 \%$ et $62,7 \%$ ).

Le taux de satisfaction par rapport aux heures de rendezvous que fixent les prestataires est élevé dans les trois districts de santé (90,6 \% à Nkongsamba, 85,9\% à Bafang et 89,5\% à Mfou). Mais, dans les deux premiers, le taux de satisfaction est plus faible chez les femmes ayant été servies à l'hôpital de district (respectivement 82,9\% et $77,4 \%)$ qu'à l'hôpital confessionnel $(93,9 \%$ et $88,4 \%)$ et dans les centres de santé (où le taux le plus faible est de 91,5\% à Nkongsamba et $84,8 \%$ à Bafang).

Le taux de satisfaction par rapport aux manières dont les prestataires recommandent les clientes à leurs collègues est aussi élevé dans les trois districts de santé (respectivement 94,7 \%, 95,2\% et $88,9 \%$ ). On observe cependant à Nkongsamba que ce taux est plus 
faible $(66,7 \%)$ dans les centres privés que dans les centres publics (100 $\%)$. On observe le même schéma lorsqu'on compare l'hôpital confessionnel (88,9 \%) à l'hôpital de district (100 \%).

\section{Conclusion et recommandations}

Si l'on en croît notamment les déclarations des enquêtées sur les attitudes des prestataires et leur degré de satisfaction, l'on peut conclure que le niveau de la qualité des soins prénatals est élevé dans les trois districts de santé. Mais, compte tenu du fait que des problèmes importants ont été observés aux niveaux structurels et du processus des soins dans notre étude précédente, ce résultat peut aussi signifier que les clientes tolèrent dans les milieux étudiés recevoir les soins de qualité moindre.

Les résultats de cette étude suggèrent aussi que la qualité des soins varie selon le milieu, le type de formation sanitaire et l'élément de la qualité considéré. En ce qui concerne l'état des ressources physiques et celui des matériels, leurs niveaux se sont avérés plus bas dans les formations sanitaires publiques que dans les privées dans les trois districts de santé étudiés. Les capacités des systèmes des soins prénatals devraient donc être renforcées dans les premières. S'agissant de la gestion, la proportion des clientes ayant déjà été rentrées sans être servies s'est avérée à Nkongsamba davantage élevée en milieu hospitalier public. Dans le même cas, les taux de satisfaction par rapport aux heures d'ouverture et à l'organisation des services se sont avérés, dans les trois districts de santé, plus faibles chez les clientes ayant fréquenté les formations sanitaires publiques que chez d'autres. Etant donné que dans les milieux étudiés, la plupart des clientes fréquentent les premières, l'augmentation de l'effectif de prestataires qui y travaillent aurait un impact positif sur le niveau de ces éléments de la qualité. L'on devrait en plus améliorer les conditions dans lesquelles les prestataires travaillent, particulièrement leurs rémunérations, car elles peuvent aussi être liées à ces éléments de la qualité.

S'agissant des relations entre prestataires et clientes, la proportion des clientes ayant perçu le temps d'attente comme étant long s'est avérée dans les trois districts de santé davantage élevée en milieu hospitalier public. La proportion des clientes ayant perçu la durée des consultations comme étant courte s'est avérée dans les districts de santé de Nkongsamba et Mfou davantage élevée dans les formations sanitaires publiques, particulièrement dans les centres de santé. Ces résultats témoignent une fois de plus de la pertinence des 
Rwenge Mburanoe: La Qualité des Soins Prénatals selon la Perspective des Clientes au Cameroun. (Districts de santé de Nkongsamba, Bafang et Mfou)

recommandations précédentes sur l'augmentation de l'effectif de prestataires et l'amélioration de leurs conditions de travail.

Dans les trois districts de santé, les formations sanitaires publiques se sont aussi avérées plus concernées que d'autres par le problème de manque de confidentialité et celui de manque de courtoisie pendant les consultations prénatales. Ce résultat suggère non seulement que ces formations sanitaires devraient être aménagées de telle manière que les soins prénatals y soient offerts dans l'intimité mais aussi que la politique d'amélioration de la qualité dans celles-ci devrait inclure cet élément et l'autre.

Dans le cas de la compétence technique des prestataires, les résultats obtenus suggèrent qu'elle devrait en priorité être améliorée dans le district de santé de Bafang. Enfin, dans le cas du dernier élément de la qualité des soins considéré, l'utilisation régulière des services, le taux de satisfaction par rapport aux heures de rendez-vous s'est avéré plus faible en milieu hospitalier public dans ce district de santé et à Nkongsamba. En revanche, dans ce dernier le taux de satisfaction par rapport aux méthodes de recommandation à d'autres spécialistes s'est avéré plus faible dans les formations privées que publiques. L'on devrait tenir compte de ces éléments dans les politiques d'amélioration de la qualité des soins pour motiver les femmes à utiliser régulièrement les services des soins prénatals dans les milieux étudiés.

\section{Bibliographie}

BENINGUISSE, G., HADDAD, S. et NIKIEMA, B. (2003) L'accessibilité culturelle: une exigence de la qualité des services et soins obstétricaux en Afrique. Quatrième Conférence Africaine sur la Population: Population et pauvreté en Afrique : relever les défis du 21 ième siècle, Tunisie, 2003.

BENINGUISSE, G. (2003) Entre tradition et modernité. Fondements sociaux de la prise en charge de la grossesse et de l'accouchement au Cameroun. L'harmattan \& Academia-Bruyant, 297p.

GUPTE, M., BANDEWAR, S. \& PISAL, H. (1999) Women's perspectives on the Quality of General and Reproductive Health 
Care : Evidence from Rural Maharashtra". In Improving Quality of Care in India's Family Welfare Programme. Eds. M. KOENIG \& M. E. KHAN. The Population Council, Inc., pp. 117-139.

HULTON, L., MATTHEWS, Z. \& STONES, R. W. (2000). $\underline{A}$ framework for the evaluation of quality of care in maternity services. University of Southampton, 2000, 72p.

LANTIS, K., GREEN, C. P. \& JOYCE, S. (2002) Providers and Quality of Care. New perspectives on quality of care, $N^{\circ} 3$, Population Council \& Population Reference Bureau, July 2002, 8p.

MURTHY, N. (1999) The quality of family welfare services in Rural Maharashtra : Insights from a client survey". In Improving Quality of Care in India's Family Welfare Programme. Eds. M. KOENIG \& M. E. KHAN. The Population Council, pp. 33-48.

RANSOM, I. et YINGER, V. (2002): Pour une maternité sans risques. Comment éliminer les obstacles aux soins, Population Reference Bureau, Juillet 2002, 36p.

THADDEUS S. et MAINE D. (1994). “Too far to walk: Maternal mortality in context". Social Science and Medicine, 38, 8, pp. 1091-1110.

VERNON, R. \& FOREIT, J. (1999) How to help clients obtain more preventive reproductive health care. International Family Planning Perspectives, Volume 25, number 4, December 1999, 200-2. 